\title{
Tacit Knowledge and College Kunqu Education
}

\author{
Guorong Ding ${ }^{1,2}$, Bin Shao ${ }^{2}$ \\ ${ }^{1}$ School of Humanity, Soochow University, Suzhou, 215123, China \\ ${ }^{2}$ School of Art, Suzhou University of Science and Technology, Suzhou, 215011, China
}

Keywords: Tacit knowledge, Kunqu, Kunqu education

\begin{abstract}
Tacit knowledge was first proposed by Polanyi. Personal knowledge is classified into explicit knowledge and tacit knowledge, and tacit knowledge is more important than explicit knowledge. The tacit knowledge in college Kunqu heritage education mainly includes: singing skills, knowledge enthusiasm and sustenance of beliefs, etc.
\end{abstract}

\section{Introduction}

Kunqu as the oldest mature art form in Chinese opera has an outstanding literary, artistic, historical and cultural value. "There are no words to describe Kunqu but 'beautiful': beautiful vocal, beautiful figure, beautiful rhetoric, integrating the beauty of music, dance and literature in one, has been through four hundred years, tempered and consummate, already reached perfection, can be called the most exquisite and perfect form in Chinese performing arts." Said by Mr. Bai Xianyong. But after Kunqu reached its most perfect artistic peak, as the saying "one dynasty had its victory" (Jiao Xun in Qing Dynasty), "One dynasty had its own literature" (Wang Guowei), Kunqu inevitably tended to decline. Given the outstanding historical and cultural heritage value of Kunqu, many intellectuals regarded the heritage of Kunqu as a great career for continuation of Chinese culture. In 1905, Qu theory master Wu Mei was teaching at Soochow University, "Teaching music, bringing flute into classroom" (Tang Guizhang Shortened Form Of Lily Magnolia Flowers • For Teacher Qu centenary birthday"), for the first time introduced Kunqu into higher education classroom. Then two or three decades, Mr. Wu Mei taught at several universities in Beijing, Nanjing and other places, dedicated the song to learn, to teach himself the flute sing opera, training a group of songwriters scholar and educator. Kunqu college education so far has been a century.

In twentieth century, the same strain of traditional Chinese culture emerged a fault. Kunqu which was originally a highbrow art was facing an endangered situation because double deletions actors and the audience. Fortunately, since this century, Kunqu especially after by UNESCO announced as the first batch of "Human Oral and Intangible Heritage of Humanity", more and more attention by the public. With the youth version of Kunqu "Peony Pavilion" tour and various universities at home and abroad to promote Kunqu into education and other campus communication activities, students become more contacts and understanding it, about the art of Kunqu has gradually opened Liberal elective in college whereby university curriculum and extracurricular classroom together to build a campus "opera fever" a landscape, a young intellectual audience layer is gradually develop and rise.

\section{Tacit knowledge concept}

Tacit Knowledge concept was first proposed by the famous British physical chemist and philosopher Michael Polanyi (1891-1976). Polanyi believed that the formation of knowledge depends on the knower of individual activities. In order to clarify the idea that "Knowledge is personal", Polanyi put forward the definition of tacit knowledge in the book Personal Knowledge (1958). "There are two types of human knowledge. Those usually described as knowledge, that written text, diagrams and mathematical formulas to be expressed, only one type of knowledge. And knowledge is not express, as we work actions in the have the knowledge, is another kind of knowledge. "He called the former explicit knowledge, the latter known as tacit knowledge. As can be seen, explicit knowledge is the knowledge can be expressed in a variety of symbols, and tacit knowledge means 
that we know that it is difficult to explain in words of knowledge. Most people think explicit that knowledge is the whole of human knowledge; in fact it is just tip of the iceberg on the water, while tacit knowledge is hidden in underwater macro most.

\section{Tacit knowledge in college Kunqu education heritage - taking cantata as the example}

Kunqu is a comprehensive performing art integrating music, dance and literature and the knowledge involved includes a lot of tacit knowledge components. For hundreds of years, Kunqu heritage all the way down, and retained since becoming the oldest mature operas. Kunqu opera is called a "living fossil", "oral teaching" traditional way thanks to Kunqu heritage, as well as researchers and practitioners who tried to capture the spread for Kunqu Opera performed. In fact, since 400 years ago Wei Liangfu reformed Kunshan Tone began its vocal music, the official, folk are constantly engaged in large-scale scores composing, substantial achievements, such as the "North-South culmination word spectrum" and "Nashu Studio Theatrical Music"and so on. Kunqu spectral figure relatively rare, but there are also achievements of their predecessors, such as the Qing dynasty period harp hidden edition Weng "sound trial Kam ancient record," Xu Lingyun "Kunqu Opera performance gains," and so on. Around music and opera performances, more written records are scattered among the vast poems notes. In this sense, the song home for hundreds of years artists who are making individual efforts of tacit knowledge explicit. However, Kunqu opera artistic heritage such as music scores alone is not enough to learn. On opera music is concerned, previous employment record ruler spectrum, more than just record the trunk, learners need to understand the details. The so-called "Understand the theory and music comes with mouth" is tacit knowledge that is learned.

About ingredient or type of tacit knowledge, there are multiple studies. Polanyi stated that tacit knowledge and explicit knowledge constitutes personal knowledge system, and he stressed the importance of tacit knowledge. Tacit understanding is essentially an understanding, and an ability to understand, grasp and restructure experience to achieve rational control on it. Tacit powers of mind play a dominant role on all levels of human knowledge. The most important feature of tacit knowledge that it must rely on understanding of individual existence, the human body and mind is to reach tacit knowledge tools. For example Polanyi scientific therefore believes that human factors such as judgment, knowledge enthusiasm, faith, conscience, commitment and sense of responsibility, and so are personal tacit knowledge. Combined with the current existing research, based on the characteristics of the Inheritance of opera education in colleges and universities, this paper argues universities Kunqu heritage education in tacit knowledge mainly in the following aspects:

\section{Singing skills}

Singing skill is the knowledge of know-how aspect which belongs to tacit knowledge. "If you want to understand Qu theory, you must first sing" (Wu Mei). Universities opera singing practices often take the form of a cappella. A cappella, "better than the play field by the percussion of the potential, all to purge elegance, Kiyotoshi warm" (Wei Liangfu in Ming Dynasty "Qu theory"). Qing, first to perform without makeup, second is not noisy accompaniment, or only one flute, or in combination with clappers beat, singing songs easy to highlight.

In terms of a cappella, Wei Liangfu's Qu Theory states: "There are three tricks of Qu: first clear words, second pure tone and third accurate allegro.” Therefore, "clear words", "pure tone” and "accurate allegro" are called example "three tricks” of a cappella.

"Singing in accordance with words" is the basic law for Kunqu singing. Therefore "clear words" is the first condition of singing. For the tone and voicing yin yang of the word, the ancients had developed a specification and widely spread, such as rhyme book "Central Plains phonology", "Hongwu Rhymes", "Phonology Selection" and so on. "Pentameter with tones based, tones it should not be, then carry the waste pentameter. Level up into, individually elegant, service was Chiang Kai-shek, as struggling contradictory or incorrect tone from good, although with linger, undesirable 
end its or make twist rising tone toned, mixed cropping Falling Tone, delivery unknown, are doing it to show off the chamber, the knower of the debate. "(Wei Liangfu Qu Thoery)

For words, we have learned to distinguish tones; however, it is not easy to sing. Shen Kuo in Song stated in Dream Pool Essays Volume 5: an important law of Chinese Art of Singing: "No words in the sound; have sound in the word." Zong Baihua summarized the experience of ancients singing art, believed that the essence of "No words in the sound; have sound in the word" is made the word melt, the word that the head of abdominal anatomy tail of three parts, into the 'chamber'. 'Character' is denied, but the 'word' of the content in singing but has been fully expressed. There is both the expression of the content, but also the art of charm. "

But to do Kunqu opera "pure tone" is more difficult. Tone grid is now commonly used to characterize the chamber opera and singing words rhyme, sound and emotion-related programs and singing mouth cavity line method. Chamber opera tunes grid including Word format (such as ooh chamber, stacked chamber, etc.) and American tunes grid (such as shake chamber, the chamber slide, take the chamber, etc.). Shen Chongsui "Qu Information" for four tunes sung Kun grid are discussed in detail. The overall specification is: level up into four tones, the "falling tone when singing, humming when rising tone, flat, Tone and when appropriate, its level, can not make the mix" (Shen Chongsui "Qu Information" quoted Shen Xi’s statement); in the same tone, the female voice singing, humming sound positive.

Kunqu sing mouth act regulates the sound of singing different words, rich tunes retouching, conducive to the performance of the song feeling. But for more than ten kinds of mouth method, the scores are usually marked only a few species, such as stacked chamber, shake chamber, excluded cavity, broken cavity, etc., the other chamber is generally not marked as olives and the like. These details are not explicitly recorded, and only rely on the mouth and heart mentoring was able to spread. In fact, it marked the opening Qinshou law also required in order to properly get.

It is these hundreds of years of heritage Kun sing mouth method, making opera with a pet Shen Sui "Qu Information" of the "Call mill, beat endure cold, the sound level up into the wan Association, the word is the first belly Bi Rong tail of uniform power cut deep, gas-free fireworks, Kai Kouqing round, radio pure fine "art of character.

Similarly, it is not easy to achieve "accurate allegro". Allegro, namely shoot, rhythm. There are six Kunqu allegros: a plate three very slow with gifts board, a board of three Adagio, the speed of stiff board with gifts, Allegro's stiff, fast flowing water have board eyeless and freedom rubato. Several chamber opera has a shot, the number of revolutions of the characteristics of a cavity, you must master the beat and tempo at the beginning to learn to sing. Mannequin an often pat a hundred times, it requires rhythm correct, skillful and then stop.

For opera music, in addition to external rhythms, but also comprises a subtle internal rhythm, the "core plate." External rhythm is the basic rhythm of the music and inertia, "heart plate" is the opera song to sing the highest aesthetic value and unique charm lies. Only internal rhythm opera singing has a keen perception of the rhythm just the right sense of proportion, it will have a specific external rhythmic rendering. In fact, the rhythm changes nuanced only embodies the true meaning of the existence of opera music. When we sang "delicate silk sunny courtyard busy blowing, shaking Yang Chun, such as line," you can feel the charm and it's hard to explain in words to express the heart of the plate.

\section{Knowledge enthusiasm}

Polanyi in his Personal knowledge dedicated a chapter to the book knowledge enthusiasm. He believed that no scientist purely scientific interest, no scientist full participation passion, no scientist put their whole life bit by bit, like put a bet, any scientific discovery has great significance is impossible. For Kunqu is concerned, for the improvement of Kunshan tone, Wei Liangfu put life energy. According to Yu Huai’s "Jichang Garden Songs": "Nanqu began from Kunshan Wei Liangfu, Liangfu learnt north music at the beginning, constraints on who is the king of Friends mountain retreat Lou heart Nanqu, footprints do not go downstairs by decade. When it is, the curvature of straight south unintentionally cause. Liangfu special throat bet tune of a new sound, Ji Xu compete, 
voicing the number one under this palace. Retrieved from tooth lips, clever drop-down change, and constant to help its desolate appearance deep. Lou heart Nanqu, footprints were not downstairs decade "this enthusiasm for knowledge, so reform Kunshan cavity Wei Liangfu the road to success.

\section{Sustenance of beliefs}

Polanyi believes that all knowledge has a tacit premise, contains a set of unexpressed, sometimes it is impossible to express the assumptions and beliefs. He will know the tacit premise called sustenance framework. Sustenance framework as people raising activities can not break free of the preset framework, including the awareness of all kinds of activity-dependent beliefs, also contains all liability for possession of personal beliefs have. Therefore, sustenance is holding faith behavior, but also has behavioral responsibility.

For Kunqu, Wei Liangfu believes that "there are two song not identified: those who do not know the sound cannot be identified with them; a bad person, cannot be identified with them." And when the duet "Elegant purge, clean and warm" and enjoy the time, "listening to music cannot be noisy, listen to his articulation, orderliness, through the chamber properly before Bianqi Clumsy. guttural not clear, it was hit section extol. "These are the existence of implicit beliefs about opera, that opera is the opera of the literati, It is a refined culture. So people do not need to learn Kunqu know people do not like the sound of opera people to distinguish, this is a faith; while Cantata "Elegant purge, clean and warm" reflects the conviction and responsibility; not noisy while listening carefully listen to his "three" skills is appropriate, it is also a responsibility. Whether singing or enjoy singing other people's behavior should keep literati unique temperament, this temperament likelihood elegant orchid in general.

"Kunqu has been criticized by people for it is too high to be popular, but I do not think so. I think in the twentieth century, Chinese people's temperament had become too rough, and it is subject to enlighten with Kunqu this exquisite culture." Says Mr. Bai Xianyong. "If there is no young audience, Kunqu cannot be developed in the future." Mr. Bai Xianyong believes, "the most important college students group in youth, have relatively higher culture and art appreciation capability, they should become pillar group of Kunqu."

Above limited by the space and the authors' level, just taking clear Qu singing as an example outlined the major tacit knowledge in Kunqu education. For the teaching of tacit knowledge and others, it needs to be further studied.

\section{Acknowledgments}

This paper belongs to general social sciences research project of Ministry of Education Kunqu Intangible Cultural Heritage Image Database System Construction (11YJAZH076), and a project of science of education $12^{\text {th }}$ Five-Year Plan in Jiangsu Province Tacit knowledge research in Kunqu Opera Inheritance (T-c/2013/015).

\section{References}

[1] Wu Xinlei. Thesaurus of Chinese Kunqu . Nanjing: Publishing House of Nanjing University, 2002.5.

[2] Chen Duo, Ye Changhai. Selected interpretation for China's opera theories in past dynasties . Shanghai: Shanghai Ancient Books Publishing House, 2010.6.

[3] Zhou Qin. Suzhou Kunqu Opera. Suzhou: Publishing House of Suzhou University, 2004.4.

[4] (UK)Michael Polanyi. Personal Knowledge: Towards a Post-Critical Philosophy . Translated by Xu Zemin. Guiyang: Guangzhou People’s Publishing House, 2000.10.

[5] Yu Zhenhua. Human knowledge's tacit dimension . Beijing: Publishing House of Peking University, 2012.9. 
[6] Li Baihe. To know the reestablishment of idea on tacit dimension: Polanyi’s tacit epistemology . Beijing: China Social Sciences Publishing House, 2009.11.

[7] Bai Xianyong: Bai Xianyong’s comments on Kunqu, Guangxi Normal University Press 2004 Edition, P. 5.

[8] Chen Wangheng: China ancient history of aesthetics, Hunan Educational Press, 1998 Edition, P 124.

[9] Chen Duo, Ye Changhai: Selected interpretation for China's opera theories in past dynasties, Shanghai Ancient Books Publishing House, 2010 Edition, P359.

[10]Zong Baihua: On aesthetics, Shanghai People’s Publishing House, 1981 Edition, P60.

[11]Zhou Qin: Suzhou Kunqu Opera, Suzhou University Press, 2004 Edition, P41.

[12]Lin Huiru from Danyang: Anecdotes in Ming, Zhonghua Book Company, 1919 Edition, P28.

[13]Radio Television Hongkong: Telefilm Outstanding ethnic Chinese series - Bai Xianyong. 